\title{
4. Placenames as a guide to language distribution in the Upper Hunter, and the landnám problem in Australian toponomastics $^{1}$
}

\author{
Jim Wafer \\ University of Newcastle
}

\section{Introduction}

The question of what language or languages was or were spoken in the Upper Hunter region of New South Wales (NSW) is vexed. The NSW Aboriginal Languages Research and Resource Centre's current draft 'Map of NSW Aboriginal languages' ${ }^{2}$ leaves the Upper Hunter blank. In earlier attempts to identify the languages spoken in this region, from the coastal areas occupied by 'Awabakal' and 'Worimi' to the headwaters of the Hunter in the Great Dividing Range, Tindale (1974: 193 and map) filled the space with a language he called 'Geawegal', and the Central Mapping Authority ('CMA') of NSW (1987) and Horton (1996) followed suit.

Brayshaw, on the other hand, analyses a number of historical documents that suggest a long-standing connection between speakers of 'Kamilaroi' and the upper Hunter (1986: 38-42), 'as far south as Wollombi Brook'3 (1986: 41). There seems to be little disagreement that the Kamilaroi occupied the headwaters of the Hunter (Tindale 1974: 194 and map) and the area around Murrurundi, close to the watershed. Further down the Hunter Valley, the literature often depicts the Kamilaroi as intruders. In relation specifically to the Geawegal, Fison and Howitt say that the latter 'were always in dread of war with the Kamilaroi, who intruded down the heads of the Hunter across from Talbragar ${ }^{4}$ to the Munmurra waters, ${ }^{5}$ and even occasionally made raids as far as Jerry's Plains ${ }^{6}$ (1880: 279).

\footnotetext{
1 I am indebted to Amanda Lissarrague, John Giacon, David Nash and Stephen Wye for helpful correspondence and conversations pertaining to this article.

2 http://ab-ed.boardofstudies.nsw.edu.au/bosImg_window.cfm?objectid=03540C9F-F074-1B07416F6CDE53A110F7> (accessed 6 April 2014).

3 Which joins the Hunter just upstream from Singleton. Nonetheless, Brayshaw's map (1986: 39), probably following Tindale, allocates the Upper Hunter to speakers of Geawegal.

4 The Talbragar River rises in the Warung State Forest (between Murrurundi and Cassilis) and flows into the Macquarie River near Dubbo. (See http://www.gnb.nsw.gov.au/place_naming/placename_search/ extract?id=TRlpXtsETR, accessed 6 April 2014.)

5 The Munmurra River is a tributary of the Goulburn that rises near Breeza Lookout and flows through Cassilis.

6 Between Denman and Singleton.
} 
The present article attempts to throw some light on this matter, mainly through an analysis of placenames from the region, and then investigates some of the difficulties posed by landnám ${ }^{7}$ in the context of Aboriginal placenames. But I begin with an investigation of the term 'Geawegal', since it has been given such prominence by the two scholars (Tindale and Horton) who have been most influential in determining the course of Aboriginal language cartography.

\section{Geawegal}

The use of 'Geawegal' as the name for a social and linguistic grouping originated with Fison and Howitt's account of 'The Geawe-gal Tribe' (Appendix G of their Kamilaroi and Kurnai, 1880: 279-284), based on information provided by G.W. Rusden. ${ }^{8}$ This short study contains only six morphemes associated specifically with Geawegal. These include geawe, the word for 'no', from which the language derives its name.

The negligible size of this body of data makes the linguistic classification of Geawegal difficult. Tindale assumed that it was related to Worimi. He wrote as follows: 'Affiliated with the coastal Worimi. The grammar and vocabulary published by Hale (1845), following Threlkeld (1834), relates principally to this tribe. The ascription of it to Kamilaroi by Hale is an unexplained error' (Tindale 1974: 193).

Tindale mentions two dialect names here: 'Worimi' and 'Kamilaroi'. In a more contemporary orthography these would be 'Warrimay' and 'Gamilaraay'. Warrimay is a dialect of what I call the 'Lower North Coast' language (LNC), which includes as well the dialect known as 'Gringai' ('Guringay'). Gamilaraay is a dialect of what I call the 'Darling Tributaries' language (DT).

There is a third language that will be pertinent to the discussion here, namely, the Hunter River-Lake Macquarie language (HRLM). This is sometimes called 'Awabakal', although this name (invented by John Fraser 1892: v) is probably best reserved for the northern coastal dialect. The language includes other dialects as well, such as 'Wonnarua' ('Wanarruwa').

Tindale is correct that Hale's use of the name 'Kāmilarai' in reference to his published language material is a mistake, since it clearly pertains to the Hunter River-Lake Macquarie language. But he is wrong in his assumption that the

7 I shall consider the term in more detail at the appropriate point in this paper. Here, a simplified definition will have to suffice: 'the cultural adoption of a landscape by immigrants to an area'.

8 The material in Fison and Howitt's Appendix G summarises data about the Geawegal that are scattered throughout Howitt's later publication, The Native Tribes of South-East Australia (1904). Howitt's 1904 work adds nothing new. 
linguistic work of Hale and Threlkeld refers to Geawegal, unless he is using this name as a general term for HRLM. This seems unlikely, given that he says the Geawegal are 'affiliated with the coastal Worimi'. In a similar vein, in his entry for 'Worimi', Tindale writes that 'Threlkeld's 1834 grammar is principally from the inland Geawegal with whom [the Worimi] were affiliated' (1974: 201).

Because of the influence of Tindale's work, it is worth untangling some of the strands of his argument. It is true that Horatio Hale visited Lancelot Threlkeld (in 1839), and, on the basis of his interpretation of Threlkeld's material, wrote a vocabulary and grammar that he called 'Kāmilarai' (Hale 1846: 482 and passim). As noted above, Tindale is right that such an ascription was an error on Hale's part. But I cannot find any reason why Tindale would attribute Hale's material (and the Threlkeld data on which it was based) to the 'inland Geawegal'. ${ }^{9}$

Threlkeld himself did not give any Indigenous name for the language he wrote about, but instead used the following formula (or variations of it): 'the language, as spoken by the Aborigines, in the vicinity of Hunter's River, Lake Macquarie etc., New South Wales' (1834: iii). The only informant named (1834: ix) is M'Gill (Biraban), who is known to have been a native of the Lake Macquarie district. Threlkeld does mention that his own son had visited 'the higher districts of Hunter's River', but only in order to say that the language spoken there did not appear to be mutually intelligible with the (coastal) dialect of the language spoken by himself and, evidently, by his son (1834: x). So there is no evidence, linguistic or otherwise, to suggest that the language described in Threlkeld's work of 1834 does not pertain to the same variety he wrote about in his other works - that is, the dialect spoken at Lake Macquarie and the Lower Hunter rather than to an inland variety (cf. Oppliger 1984: 1-3; Lissarrague 2006: 7-15).

Tindale's mention of the Threlkeld grammar under his entry for 'Worimi' suggests he believed that this grammar described the language spoken by the Worimi, or at least a closely related dialect - in other words, that HRLM and Worimi were dialects of the same language. Amanda Lissarrague and I have analysed in some detail the likely historical reasons for this error, and attempted to show that the Lower North Coast language (of which Worimi is a dialect) and HRLM are separate languages (Wafer and Lissarrague 2011: 149-150). For present purposes, it is sufficient to say that a comparison of the verb and pronoun paradigms of LNC and HRLM provides enough evidence to define them as distinct.

But is Geawegal related to either of these languages? Or is it perhaps, like Gamilaraay, a dialect of the Darling Tributaries language?

9 The only relevant source appears to be Enright, who says that 'the Geawegal was merely a horde, and a part of the Worimi tribe' (Enright 1932: 75). But this is not a reason to attribute the Threlkeld material to Geawegal. 
Indigenous and Minority Placenames

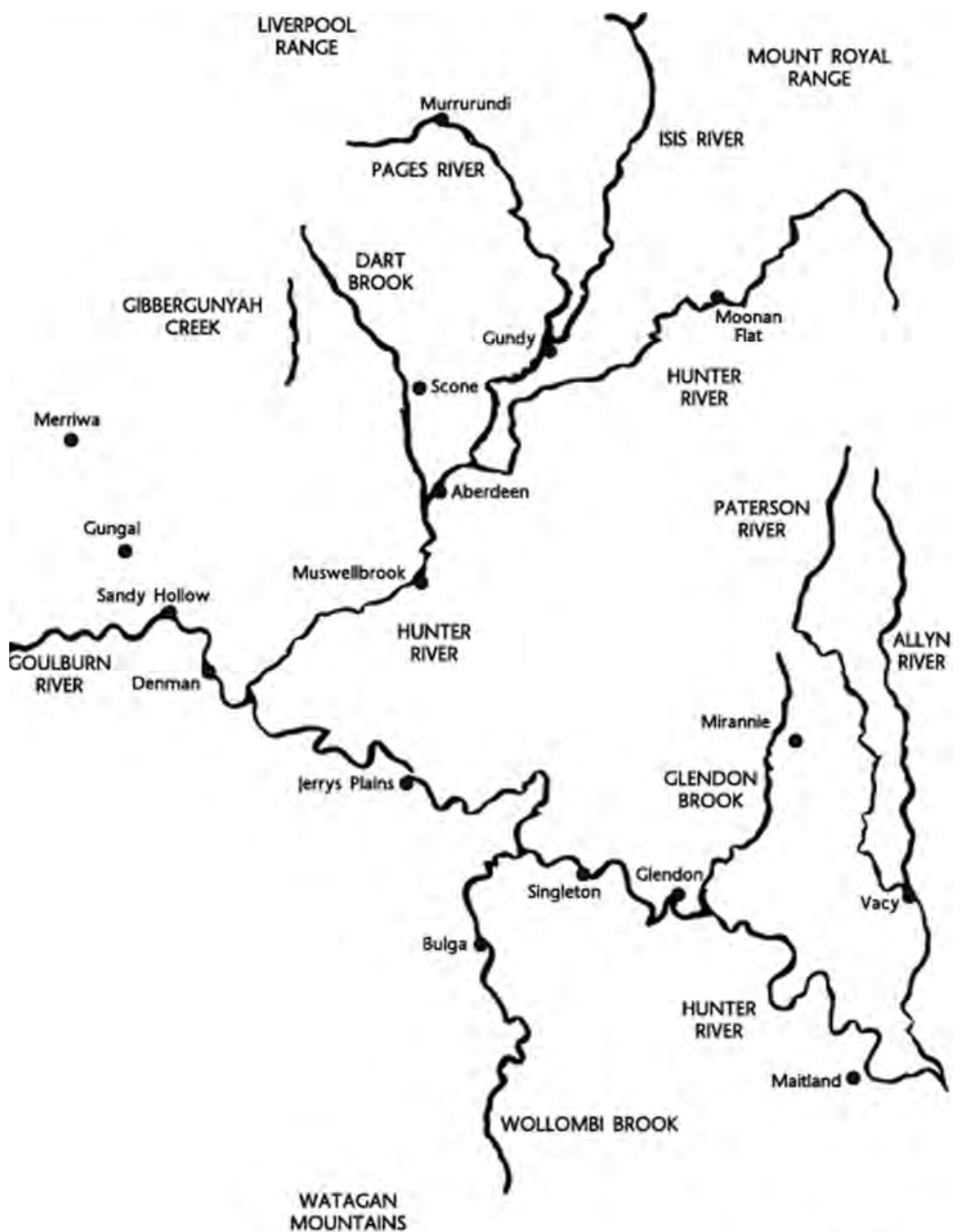

LAKE MACQUARIE

53

Map 1: Map of the Upper Hunter, with placenames mentioned in the text. Source: The author. 
As mentioned above, Fison and Howitt's account of 'The Geawe-gal Tribe' contains six morphemes from the Geawegal dialect. These include geawe, the word for 'no', from which the language derives its name. Kayaway is one of several alternative negative forms in HRLM (Lissarrague 2006: 98, 117), and is not used in LNC, where the common negative particle is gurayn (Lissarrague 2010: 238). Fison and Howitt mention two other items of vocabulary, namely koradji, 'wizard, medicine man' (1880: 281) and murramai, 'rock crystal' (1880: 283), both of which are also found in HRLM. ${ }^{10}$ But the former has a cognate in Gamilaraay (maarama, 'stone'; see Ash, Giacon and Lissarrague 2003: 104) and the latter a cognate in LNC (giraatji, 'clever man'; see Lissarrague 2010: 229).

In addition, Fison and Howitt's informant, G.W. Rusden, used one Indigenous word, with a gloss, in the notes to his epic poem 'Moyarra'. This word he rendered as barracun and glossed as 'boomerang' (Rusden 1891: 51). ${ }^{11}$ The same word occurs in both HRLM (parrkan, Lissarrague 2006: 131) and LNC (barrgan, Lissarrague 2010: 180).

Further, the dialect name 'Geawegal' (which I shall spell from here on as 'Kayawaykal') contains the derivational suffix -kal, which Lissarrague has described as the 'belonging' suffix in HRLM (2006: 62-63). ${ }^{12}$ But this suffix also occurs in LNC. ${ }^{13}$ According to Fison and Howitt (1880: 280), Kayawaykal had, in addition, words for the 'class divisions' Yippai (Yipay) and Kombo (Kampu). ${ }^{14}$ Among the speakers of the HRLM dialects, the Wanarruwa also had these class divisions (Fawcett 1898: 180), technically known as 'sections', and the speakers of the eastern dialect ('Awabakal') did not. These names have cognates in the Darling Tributaries language (Howitt 1904: 104; Ash, Giacon and Lissarrague 2003: 10-12). The western dialect of LNC (Guringay) also used section names of this type (the 'Kamilaroi type'), but, according to Howitt, in a somewhat modified form (1904: 266-267). ${ }^{15}$

This is admittedly a very small body of language material on which to make a case. What we can say about it, in summary, is that all of the seven morphemes

10 Lissarrague has reconstructed these as maRamay maRama (pers. comm.) and kuratji (2006: 120).

11 It is worth noting Fison and Howitt's observation that Rusden 'was identified with [the Geawegal 'tribe'], and spoke the language as a youth' (1880: 279).

12 As Lissarrague says, the name 'Kayawaykal' 'is composed of an HRLM word for "no", kayaway, and the belonging suffix -kal' (2006: 13).

13 In the orthography Lissarrague uses for LNC, this suffix is spelt as -gal. Its meaning is similar to that of -kal in HRLM, but in LNC Lissarrague glosses it as 'type [of person]' (2010: 47-48).

14 It is unclear why Rusden recorded only two names for the 'class divisions', where the neighbouring languages that use cognate terms have four. (There are usually four terms for males, and four corresponding terms for females.) Rusden himself admits that he could 'not recollect all their class divisions' (Fison and Howitt 1880: 280), so it seems more likely to be a result of the recorder's faulty memory than a reduction of the four 'section' terms to two.

15 'Most of the Gringai were named Kumbo, but there were some Ipai, Kubbi and Murri among them' (Howitt 1904: 266). Howitt goes on to observe what he regards as a 'complete breaking down of the old Kamilaroi system' among the Gringai (1904: 267). 
that occur in Geawegal are found in at least some of the other dialects of HRLM. Moreover, while there are cognates in some of the surrounding non-HRLM languages, the cognate count is never higher than five out of seven items. ${ }^{16}$ This suggests that, on the balance of probabilities, Kayawaykal was a dialect of HRLM, and not a dialect of one of the neighbouring languages, such as the LNC or the Darling Tributaries language.

\section{Locating Geawegal}

Because the corpus of linguistic data associated explicitly with Kayawaykal is so restricted, I have attempted to compensate for this lack by analysing sources which, while not specified as Kayawaykal, come from the area in which Kayawaykal was supposedly spoken. But the extent of this area is not unambiguous, since Fison and Howitt (the earliest source) and Tindale give different accounts.

According to Fison and Howitt, 'the territory claimed by them [the Kayawaykal] may be defined as being part of the valley of the Hunter River extending to each lateral watershed, and from twenty-five to thirty miles along the valley on each side of Glendon. [Glendon is a short distance south-east of Singleton.] These aborigines spoke the language of, and intermarried with, those of Maitland. Less frequently with those of the Patterson [sic] River, and rarely with those of Muswell Brook' (1880: 279).

This account is broadly contradicted by Tindale (1974: 193 and map), who provides this version of the location of the Kayawaykal: 'Northern tributaries of the Hunter River to Murrurundi; at Muswellbrook, Aberdeen, Scone, and Mount Royal Range' ${ }^{17}$ In other words, Tindale places the Kayawaykal quite a long distance further to the north-west than Fison and Howitt.

There are two manuscript documents that have the potential to adjudicate between these contradictory accounts. One pertains to a location reasonably close to that specified by Fison and Howitt, and another to a region well within the area specified by Tindale. The first of these (Anonymous ca 1845) is a wordlist collected at the Allyn River, which, at its closest point to Glendon (at its confluence with the Paterson River at Vacy) is roughly $40 \mathrm{~km}$ (25 miles) to the east. Lissarrague (2010: 12) treats the words in this list as belonging to the Lower North Coast language, and the available evidence supports this view

16 The cognate count appears to be five out of seven items in LNC, and three out of seven items in Gamilaraay.

17 See also the CMA (1987) map, the Horton map (1996), and Brayshaw's map (1986: 39). 
(see Wafer and Lissarrague 2008: 165, 170). According to Miller (1985: 246, 256-257), the people of the Allyn River were the 'Gringai'; and these Guringay spoke a dialect of LNC.

The presence of LNC speakers at the Allyn is not fundamentally inconsistent with Fison and Howitt's claim that the land of the Kayawaykal extended 'to each lateral watershed [of the Hunter River], and from twenty-five to thirty miles along the valley on each side of Glendon' (1880: 279). If Glendon was at the centre of their territory, this probably means they also inhabited Glendon Brook, which flows from the southern foothills of the Mount Royal Range to join the Hunter just downstream from Glendon. In the direction of the opposite watershed, their domain could well have extended along Wollombi Brook, which rises in the Watagan Mountains and flows past Bulga, to join the Hunter upstream from Singleton.

It is worth noting in this regard that 'Bulga' is probably derived from the HRLM word palkarr, 'mountain'. ${ }^{18}$ The name 'Watagan' is not so easy to analyse, but possibly includes the HRLM derivational suffix - kan (Lissarrague 2006: 65). ${ }^{19}$ If the Kayawaykal inhabited the riverine margins of the Hunter and the tributaries converging on the region around Glendon, their range probably fell a fair way short of the Allyn. Thus the only evidence that contradicts the location given by Fison and Howitt is Tindale's account of Geawegal territory, which is centred on Scone.

\section{The Scone list}

It is for this reason that the second manuscript document, which pertains to Scone and its environs, merits a more extended treatment. The manuscript was a response to a survey carried out by the Anthropological Society of Australasia in 1899. A circular was sent to police stations throughout New South Wales, with a request for 'Native Names of places with their meanings'. Many of the responses were hasty and superficial, but some, including the return from Scone, included not just placenames that had been adopted by the settler culture, but a more

\footnotetext{
18 Lissarrague (2006: 130) reconstructs this word as palkirr; but it is balgarr in LNC (Lissarrague 2010: 175). Moreover, Miller (1887: 335) records the (Wanarruwa) expression bulgargoba, 'towards the south', which I interpret as palkarr-kupa, 'mountain' + genitive).

19 The morphemes palkarr and -kan also occur in LNC (albeit spelt differently). Nonetheless, I assume that the relevant placenames are derived from HRLM, since there is no evidence that the territory of LNC speakers extended this far west. In the direction of the opposite watershed, there is another placename of likely Indigenous origin that could have some relevance to the issues discussed in this paper, namely, 'Mirannie', which designates a locality close to the headwaters of Glendon Brook. But unfortunately there is no obvious way of analysing this word in any of the three languages discussed in this paper.
} 
extensive list of vocabulary items as well. At least some, if not all, of these were probably Indigenous placenames, or at least place-descriptors, that were never incorporated into settler geography.

Here is the list, with its covering letter. ${ }^{20}$

Anthropological Society of Australasia

Police Station Scone

16th Oct 1899

Vide Anthropological Society's circular of the 15/8/99

Senior Sergeant Coady begs leave to return attached list with the Aboriginal names of places etc, and their meanings[.] He has left manuscript lists with several of the oldest residents of this district, who are said to have a good knowledge of the aboriginal language, but some have not returned their lists, when they do so, he will forward them on.

John Coady S.S.

The Supt of Police

West Maitland

20 My copy of this list comes from the version digitised by the Geographical Names Board of NSW and copied as PDF and TIFF files to CD. The contents of the CD are divided into five folders, corresponding to the rolls of the microfilm version. The Scone list is included in roll 1 , and the frame numbers are 010328 and 010329 (the list itself) and 010330 (the covering letter). I have included it in the list of references under 'Coady'. 
Table 1: Scone wordlist transcribed from Coady (1899).

\begin{tabular}{|c|c|c|c|}
\hline $\begin{array}{l}\text { Name of } \\
\text { Place }{ }^{\text {A }} \\
{ }^{*} \text { Miketymulga } \\
\text { Bibil }\end{array}$ & $\begin{array}{l}\text { Meaning or reason } \\
\text { it was given } \\
\text { Tree struck by } \\
\text { lightning } \\
\text { White leaved box } \\
\text { tree }\end{array}$ & Possible etymology & Location \\
\hline $\begin{array}{l}\text { Moonbil (now } \\
\text { Aberdeen) }\end{array}$ & Green head Ant & $\begin{array}{l}\text { D muwan, 'greenhead } \\
\text { ant' (A 117) + } \\
\text {-bil,'with a lot of' (A } \\
\text { 38) }\end{array}$ & Aberdeen \\
\hline Tamori & Pigeon ground & $\begin{array}{l}\text { D dhamarr, 'bronzewing } \\
\text { pigeon' (A 55) + } \\
\text {-araay, 'with, having' } \\
\text { (A 25) }\end{array}$ & \\
\hline Dilgoor & $\begin{array}{l}\text { Junction of Page \& } \\
\text { Hunter Rivers }\end{array}$ & $\begin{array}{l}\text { ? D dhiil, 'wilga'; 'tail' } \\
\text { (A 61) + guraarr, 'long; } \\
\text { tall' (A 97) }\end{array}$ & $\begin{array}{l}\text { Junction of Pages } \\
\text { River and Hunter } \\
\text { River (short } \\
\text { distance north-west } \\
\text { of Aberdeen) }\end{array}$ \\
\hline Goonindri & $\begin{array}{l}\text { Water coming over } \\
\text { Rocks }\end{array}$ & $\begin{array}{l}\text { D gungan, 'water' (A } \\
\text { 96) + -baraay,'with, } \\
\text { having' (A 33) }\end{array}$ & \\
\hline Booloomin & Apple Tree & $\begin{array}{l}\text { D bulamin, 'angophora } \\
\text { (appletree)' (A 44) }\end{array}$ & \\
\hline Booroobilla & Wild Turkey & $\begin{array}{l}\text { ?? D burrgulbiyan, } \\
\text { 'turkey bush' (A 48) }\end{array}$ & \\
\hline Downgimbie & Mussel ground & $\begin{array}{l}\text { D dhawun, 'earth, } \\
\text { ground' (A 59) + } \\
\text { giinbay, 'small mussel' } \\
\text { (A 188) }\end{array}$ & Muswellbrook? ${ }^{B}$ \\
\hline Boombil & Native Camping ground & $\begin{array}{l}\text { D bunbul, 'meeting } \\
\text { place' (A 46) }\end{array}$ & \\
\hline Gillebri & Moonshine & $\begin{array}{l}\text { D gilay, 'moon' (A } \\
88)+- \text { baraay, 'with, } \\
\text { having' (A 33) }\end{array}$ & \\
\hline Beroobri & Deep Water Hole & $\begin{array}{l}\text { D biruubaraay, 'hollow' } \\
\text { (A 42) }\end{array}$ & \\
\hline Myoon Myooan & $\begin{array}{l}\text { Red and Yellow Stones } \\
\text { Natives used for } \\
\text { Tattooing }\end{array}$ & $\begin{array}{l}\text { ?? D maayama, 'stone' } \\
\text { (A 105) }\end{array}$ & \\
\hline Goona Goona & Yellow stones for ditto & $\begin{array}{l}\text { H kunakuna, 'stone... } \\
\text { used for paint' (LO6 } \\
\text { 119) }\end{array}$ & \\
\hline Gullingoorar & Long Water Hole & $\begin{array}{l}\text { D gali, 'water' (A 76) } \\
+ \text { guraarr, 'long' (A 97) }\end{array}$ & \\
\hline
\end{tabular}




\begin{tabular}{|c|c|c|c|}
\hline $\begin{array}{l}\text { Name of } \\
\text { Place }{ }^{\text {A }} \\
{ }^{*} \text { Miketymulga } \\
\text { Bibil }\end{array}$ & $\begin{array}{l}\text { Meaning or reason } \\
\text { it was given } \\
\text { Tree struck by } \\
\text { lightning } \\
\text { White leaved box } \\
\text { tree }\end{array}$ & Possible etymology & Location \\
\hline Danboor & Clear Land, no water & $\begin{array}{l}\text { D dhawun, 'earth, } \\
\text { ground' (A 59) + ?? } \\
\text { bura, 'bone' (A 47) } \\
\end{array}$ & \\
\hline Goombaban & Dusty soil & $\begin{array}{l}\text { D gumba, 'flinty' (A } \\
\text { 94) + -baa, 'place of' } \\
\text { (A 25) }\end{array}$ & \\
\hline Bragar & Sparrow Hawk & $\begin{array}{l}\text { D biyaagaarr, 'brown } \\
\text { falcon' (A 42) }\end{array}$ & \\
\hline Goodangoodan & Hard substance & $\begin{array}{l}\text { ?? L gurung, 'solid' } \\
\text { (L10 240) }\end{array}$ & \\
\hline Kingimbon & Rocks & $\begin{array}{l}\text { ?? D giin.gii, 'froth' (A } \\
\text { 87) Cf. Gingamboon } \\
\text { (below) }\end{array}$ & \\
\hline Yowri & Close to; Near & $\begin{array}{l}\text { ?? D yawa, 'to track' } \\
\text { (A 152) }\end{array}$ & \\
\hline Moonan & Difficult to accomplish & $\begin{array}{l}\text { ?? D muurr, 'blocked; } \\
\text { blunt' (A 117) }\end{array}$ & $\begin{array}{l}\text { Moonan Flat? } \\
\text { (Upper Hunter) }\end{array}$ \\
\hline Billar & Swamp Oak Trees & $\begin{array}{l}\text { D bilaarr, 'swamp oak' } \\
\text { (A 38) }\end{array}$ & \\
\hline Naraman & A long way behind & $\begin{array}{l}\text { ? D ngaarrima, 'over } \\
\text { there' (A 120) }\end{array}$ & \\
\hline Woombrambilla & $\begin{array}{l}\text { A flat stick with a } \\
\text { hook used by Natives } \\
\text { in Spear throwing }\end{array}$ & $\begin{array}{l}\text { D wamara, 'spear } \\
\text { thrower' (A 137) + ?? } \\
\text { burranbaa, 'new' (A } \\
\text { 48); } \\
\text { H wamarr, 'woomera' } \\
\text { (L06 140); L wamarr, } \\
\text { 'woomera' (L10 268) }\end{array}$ & \\
\hline Gooloorooeybri & Bull Frogs & $\begin{array}{l}\text { ?? D yurayaa, 'frog' (A } \\
\text { 157) + - baraay, 'with, } \\
\text { having' (A 33) }\end{array}$ & \\
\hline Gingamboon & Hunter River & $\begin{array}{l}\text { ? D giin. gii, 'froth' (A } \\
\text { 87) Cf. Kingimbon } \\
\text { (above) }\end{array}$ & Hunter River \\
\hline Bambill & Mistletoe & $\begin{array}{l}\text { D baan, 'mistletoe' (A } \\
26)+- \text { bil, 'with a lot } \\
\text { of' (A 38) }\end{array}$ & \\
\hline Galliebarinda & Waterfall, Cascade & $\begin{array}{l}\text { D gali, 'water' (A 76) } \\
\text { + ? barra, 'to fly' (A } \\
\text { 34); H kali, 'water' } \\
\text { (L06 113) }\end{array}$ & \\
\hline Gungalla & Rushes & $\begin{array}{l}\text { H kungka, 'reed' (LO6 } \\
\text { 119) }\end{array}$ & $\begin{array}{l}\text { Gungal? (between } \\
\text { Merriwa and Sandy } \\
\text { Hollow) }\end{array}$ \\
\hline
\end{tabular}




\begin{tabular}{|c|c|c|c|}
\hline $\begin{array}{l}\text { Name of } \\
\text { Place }{ }^{\text {A }} \\
{ }^{*} \text { Miketymulga } \\
\text { Bibil }\end{array}$ & $\begin{array}{l}\text { Meaning or reason } \\
\text { it was given } \\
\text { Tree struck by } \\
\text { lightning } \\
\text { White leaved box } \\
\text { tree }\end{array}$ & Possible etymology & Location \\
\hline Yourooie & Kangaroo's Cave & $\begin{array}{l}\text { ?? D gurru, 'hole' (A } \\
\text { 98) }\end{array}$ & \\
\hline Marrawan & One hand & $\begin{array}{l}\text { D mara,'hand; } \\
\text { finger' (A 108) + } \\
\text {-wan,'prominent (big)' } \\
\text { (A 138) } \\
\text { Cf. Murrurundi (below) }\end{array}$ & \\
\hline Goolameran & Drooping Trees & $\begin{array}{l}\text { D gula, 'fork in tree' } \\
\text { (A 92) + ? mirriraa, } \\
\text { 'lignum [shrub]' (A 113) }\end{array}$ & \\
\hline Boogal & A Bush & $\begin{array}{l}\text { ? D bugarru, 'tree for } \\
\text { storing poison sticks' } \\
\text { (A 43) }\end{array}$ & \\
\hline Mill & An Eye & D mil, 'eye' (A 110) & \\
\hline Goolengdoogie & Spit Forehead & $\begin{array}{l}\text { D ngulu, 'face; } \\
\text { forehead; point [i.e. } \\
\text { spit (in the geographical } \\
\text { sense)]' + dhugaay, } \\
\text { 'small' (A 65); H ngulu, } \\
\text { 'forehead' (LO6 128); L } \\
\text { ngulu, 'forehead' (L10 } \\
\text { 261) }\end{array}$ & \\
\hline Yeraan & High Wind & $\begin{array}{l}\text { D ?? yuru, 'cloud' } \\
\text { (A 157) + -wan, } \\
\text { 'prominent (big)' (A } \\
\text { 138) }\end{array}$ & \\
\hline Goonabil & $\begin{array}{l}\text { Covered with human } \\
\text { excrement }\end{array}$ & $\begin{array}{l}\text { D guna, 'faeces' (A 95) } \\
+ \text {-bil, 'with a lot of' } \\
\text { (A 38) }\end{array}$ & [Cf. Coonamble] \\
\hline Gooran & An Emu & $\begin{array}{l}\text { ? D ganurran,'[group of] } \\
\text { emus' (A 79) }\end{array}$ & \\
\hline Gooallie & Of ugly appearance & $\begin{array}{l}\text { ?? D nguwalay, 'here, } \\
\text { (hereabouts)' (A 128) }\end{array}$ & \\
\hline Doongoorwah & $\begin{array}{l}\text { Rock running into the } \\
\text { Water }\end{array}$ & $\begin{array}{l}\text { D dhawun, 'ground' (A } \\
59)+ \text { guraarr, 'long' } \\
\text { (A 97) } \\
\end{array}$ & \\
\hline Youndayounda & A Sheet of Bark & $\begin{array}{l}\text { ?? D yuundu, 'stone } \\
\text { axe, tomahawk' (A } \\
158 \text { ) }\end{array}$ & \\
\hline Greenboon & Anything Green & $\begin{array}{l}\text { English 'green' + ? D } \\
\text { buuwan, 'black paint' } \\
\text { (A 51) }\end{array}$ & \\
\hline
\end{tabular}




\begin{tabular}{|c|c|c|c|}
\hline $\begin{array}{l}\text { Name of } \\
\text { Place }{ }^{\text {A }} \\
{ }^{*} \text { Miketymulga } \\
\text { Bibil }\end{array}$ & $\begin{array}{l}\text { Meaning or reason } \\
\text { it was given } \\
\text { Tree struck by } \\
\text { lightning } \\
\text { White leaved box } \\
\text { tree }\end{array}$ & Possible etymology & Location \\
\hline Boondool & A Shield & $\begin{array}{l}\text { D burriin, 'shield' (A } \\
48)+- \text { duul, 'small' } \\
\text { (A 68) }\end{array}$ & \\
\hline Breeal & \begin{tabular}{|l|} 
A Native Waddy for \\
fighting \\
\end{tabular} & $\begin{array}{l}\text { ? D bugu, 'fighting } \\
\text { club' (A 43) } \\
\end{array}$ & \\
\hline Marooan & Anything Good & $\begin{array}{l}\text { H marrung, 'good' (LO6 } \\
122 \text { ); } \\
\text { L marrung, 'good' (L10 } \\
248 \text { ) }\end{array}$ & \\
\hline Goolliar & White Gum Tree & $\begin{array}{l}\text { H kulay, 'tree' (LO6 } \\
\text { 118); ?? D gulabaa, } \\
\text { 'coolabah' (A 92) }\end{array}$ & \\
\hline Boonooboonoo & Kangaroo's camp & $\begin{array}{l}\text { ?? H punu, 'dust' (LO6 } \\
\text { 135); } \\
\text { ?? L bunyu, 'ashes; } \\
\text { dust' (L10 193-94); ?? } \\
\text { D banuwa, 'black soil' } \\
\text { (A 33) }\end{array}$ & $\begin{array}{l}\text { Boonoo Boonoo? } \\
\text { (north of } \\
\text { Tenterfield) }\end{array}$ \\
\hline Youndoogeedan & $\begin{array}{l}\text { Rock where blacks } \\
\text { used to sharpen } \\
\text { tomahawks }\end{array}$ & $\begin{array}{l}\text { D yuundu, 'stone axe, } \\
\text { tomahawk' (A158) + } \\
\text { ? -giirr, 'like, similar to' } \\
\text { (A 87) }\end{array}$ & \\
\hline Woolbadoon & A little plain & $\begin{array}{l}\mathrm{H} \text { wulu, 'grass' (L06 } \\
143)+-p a, \text { 'place of' } \\
\text { (L06 129) } \\
\end{array}$ & \\
\hline Youan & A clear flat & $\begin{array}{l}\text { H ngarawan, 'plain' } \\
\text { (L06 156); } \\
\text { L ngarrawan,'plains' } \\
\text { (L10 260) }\end{array}$ & \\
\hline Billanbri & A Creek with rushes & $\begin{array}{l}\text { ?? L bila, 'river' (L10 } \\
\text { 183); } \\
\text { D -baraay, 'with, } \\
\text { having' (A 33) }\end{array}$ & \\
\hline Woolamon & A high Cliff & $\begin{array}{l}\text { ?? L wuruma, 'wind' } \\
\text { (L10 277) }\end{array}$ & \\
\hline Walgooan & High wind & $\begin{array}{l}\text { ? L biwal, 'wind' (L10 } \\
\text { 187) + ? guuwak [ } \\
\text { guuwang], 'hard' (L10 } \\
\text { 241) }\end{array}$ & \\
\hline Goorarman & A long sheet of water & $\begin{array}{l}\text { ? D garragarraan, } \\
\text { 'straight river' (A 80) }\end{array}$ & \\
\hline Yeerallah & A strong current & $\begin{array}{l}\text { ? D yarrin, 'water } \\
\text { current' (A 152) }\end{array}$ & \\
\hline
\end{tabular}




\begin{tabular}{|c|c|c|c|}
\hline $\begin{array}{l}\text { Name of } \\
\text { Place }{ }^{\text {A }} \\
{ }^{*} \text { Miketymulga } \\
\text { Bibil }\end{array}$ & $\begin{array}{l}\text { Meaning or reason } \\
\text { it was given } \\
\text { Tree struck by } \\
\text { lightning } \\
\text { White leaved box } \\
\text { tree }\end{array}$ & Possible etymology & Location \\
\hline Yeroyan & $\begin{array}{l}\text { A large cave in } \\
\text { limestone Rock }\end{array}$ & $\begin{array}{l}\text { ? H yiru, 'water' (L06 } \\
\text { 146); } \\
\text { ?? L nguriyang, 'ear' } \\
\text { (L10 261) }\end{array}$ & \\
\hline Gundeerari & Birds Feathers & $\begin{array}{l}\text { D gundiirr, 'emu } \\
\text { feather' (A 96) + } \\
\text {-araay, 'with, having' } \\
\text { (A 25) }\end{array}$ & \\
\hline Woorwoor & Anything very large & $\begin{array}{l}\text { L wutu [reduplicated], } \\
\text { 'big' (L10 277) }\end{array}$ & \\
\hline Berieel & $\begin{array}{l}\text { Abounding with flaggy } \\
\text { Rocks }\end{array}$ & $\begin{array}{l}\text { ?? L birriwal, 'big' (L10 } \\
\text { 186); } \\
\text { ?? H piriyal, 'toes' (L06 } \\
\text { 133) }\end{array}$ & \\
\hline Neemin & Kurrajong Tree & $\begin{array}{l}\text { D nhimin, 'kurrajong } \\
\text { tree' (A 130) }\end{array}$ & \\
\hline Nirribingable & Swallows [sic] Nest & $\begin{array}{l}\text { [??] + H pilimalmal, } \\
\text { 'swallow' (L06 132) }\end{array}$ & \\
\hline Goorawoon & $\begin{array}{l}\text { Large Stones in the } \\
\text { Water }\end{array}$ & $\begin{array}{l}\text { D guraarr, 'tall' (A 97) } \\
+ \text { ? -wan, 'big' (A 138) }\end{array}$ & \\
\hline Yallimbilla & Two men & $\begin{array}{l}\text { D ngali, 'we two' (A } \\
\text { 121) + bulaarr,'two' } \\
\text { (A 44); } \\
\text { H ngalin, 'us two' (LO6 } \\
\text { 40) + pulawarr, 'two' } \\
\text { (cf. LO6 134) }\end{array}$ & \\
\hline Bunbibilla & An opossum cloak & $\begin{array}{l}\text { ?? D baya, clothes (A } \\
\text { 36) + ? -bil,'with a lot } \\
\text { of' (A 38) }\end{array}$ & \\
\hline Berginbah & A large Waterfall & $\begin{array}{l}\text { ? D bagay, 'creek; river' } \\
\text { (A 30) + -baa, 'place } \\
\text { of' (A 25) }\end{array}$ & \\
\hline Geengullion & An elbow & $\begin{array}{l}\text { D dhiin, 'elbow' (A 61) } \\
+ \text { ?? galiya, 'to climb' } \\
\text { (A 77) }\end{array}$ & \\
\hline Boolarie & Large lumps on Trees & $\begin{array}{l}\text { D buul, 'tree knot' (A } \\
50)+ \text {-araay, 'with, } \\
\text { having' (A 25) }\end{array}$ & \\
\hline
\end{tabular}




\begin{tabular}{|c|c|c|c|}
\hline \begin{tabular}{|l} 
Name of \\
Place A \\
${ }^{*}$ Miketymulga \\
Bibil
\end{tabular} & $\begin{array}{l}\text { Meaning or reason } \\
\text { it was given } \\
\text { Tree struck by } \\
\text { lightning } \\
\text { White leaved box } \\
\text { tree }\end{array}$ & Possible etymology & Location \\
\hline Mallegal & $\begin{array}{l}\text { A kind of timber or } \\
\text { scrub }\end{array}$ & $\begin{array}{l}\mathrm{P} \text { 'mallee' ['eucalypts } \\
\text { characteristically small } \\
\text { and having several } \\
\text { stems arising from a } \\
\text { common base' D 139] } \\
\text { + H -kal, 'pertaining to' } \\
\text { (L06 62-63); } \\
\text { + L -gal, 'type' (L10 } \\
\text { 47-48) }\end{array}$ & \\
\hline Beerubri & An aperture in Trees & $\begin{array}{l}\text { D biruu, 'hole' (A 42) + } \\
\text {-baraay, 'with, having' } \\
\text { (A 33) }\end{array}$ & \\
\hline Goorangi & Red paint & $\begin{array}{l}\text { ? D guway, 'blood' } \\
\text { (A 100) [hence } \\
\text { guwaymbarra, 'red' (A } \\
100) \text { ] }\end{array}$ & \\
\hline Guyar & An opossum & $\begin{array}{l}\text { D garrawirr, 'ringtail } \\
\text { possum' (A 81) }\end{array}$ & \\
\hline Bloombeen & A Green Parrot & $\begin{array}{l}\text { D bulunbulun, 'mulga } \\
\text { parrot; ringneck parrot' } \\
\text { (A 45) }\end{array}$ & \\
\hline Yawdool & A large hollow Stump & $\begin{array}{l}\text { D ngadhul, 'hollow tree; } \\
\text { stump' (A 120) }\end{array}$ & \\
\hline Murrurundi & Five fingers & $\begin{array}{l}\text { D mara,'hand; } \\
\text { finger' (A 108) + ?? } \\
\text {-wan,'prominent (big)' } \\
\text { (A 138) } \\
\text { [Cf. Marrawan (above)] }\end{array}$ & Murrurundi ${ }^{C}$ \\
\hline Bundadworndi & Kangaroo's playing & $\begin{array}{l}\text { D bandaarr, 'grey } \\
\text { kangaroo' (A 32) + ? } \\
\text { wuu, 'to go into [an } \\
\text { enclosed space]' (A } \\
\text { 146); } \\
\text { L bandaarr, 'wallaby' } \\
\text { (L10 176) + ? wuuna, } \\
\text { 'to leave' (L10 277); } \\
\text { H pantarr,'kangaroo' } \\
\text { (LO6 130) }\end{array}$ & \\
\hline Bareemal & A large waterfall & $\begin{array}{l}\text { ?? L barray, 'earth' } \\
\text { (L10 179) + ?? malu, } \\
\text { 'thunder' (L10 246) }\end{array}$ & \\
\hline Puen Buen & Small Stones & $\begin{array}{l}\text { ? H piwang, 'red tea- } \\
\text { tree' (LO6 134) } \\
\text { ? D buuwan } \\
\text { [reduplicated], 'black } \\
\text { paint' (A 51) }\end{array}$ & $\begin{array}{l}\text { Puen Buen (west of } \\
\text { Aberdeen) }\end{array}$ \\
\hline
\end{tabular}




\begin{tabular}{|c|c|c|c|}
\hline $\begin{array}{l}\text { Name of } \\
\text { Place }{ }^{\text {A }} \\
{ }^{*} \text { Miketymulga } \\
\text { Bibil }\end{array}$ & $\begin{array}{l}\text { Meaning or reason } \\
\text { it was given } \\
\text { Tree struck by } \\
\text { lightning } \\
\text { White leaved box } \\
\text { tree }\end{array}$ & Possible etymology & Location \\
\hline Gibbagunyah & House built of Stone & $\begin{array}{l}\mathrm{P} \text { 'gibber-gunyah', ['a } \\
\text { shallow cave used as a } \\
\text { dwelling or for shelter'] } \\
\text { (D 200) }\end{array}$ & $\begin{array}{l}\text { Gibbergunyah Creek } \\
\text { (west of Scone) }\end{array}$ \\
\hline Womulguy & Bend in the River & $\begin{array}{l}\text { ? H wampal, 'beach' } \\
\text { (L06 141)+ ? -kay, } \\
\text { 'characteristic of' (LO6 } \\
\text { 116) }\end{array}$ & \\
\hline $\begin{array}{l}\text { Conventions fo } \\
\text { Reliability } \\
\text { No question mark: } \\
\text { ? (one question ma } \\
\text { ?? (two question } \mathrm{m} \\
\text { (The question mar } \\
\text { consisting of two }\end{array}$ & $\begin{array}{l}\text { the etymology is convinci } \\
\text { ark): the etymology is plau } \\
\text { narks): the etymology is, a } \\
\text { ks - or their absence - } \mathrm{m} \\
\text { morphemes.) }\end{array}$ & $\begin{array}{l}\text { ing } \\
\text { sible } \\
\text { at best, speculative } \\
\text { ay precede either or both }\end{array}$ & parts of a word \\
\hline $\begin{array}{l}\text { Language name ab } \\
\mathrm{D}=\text { Darling Tribut } \\
\mathrm{H}=\text { Hunter River- } \\
\text { Wanarruwa [Wonn } \\
\mathrm{L}=\text { Lower North } \\
\mathrm{P}=\text { Pidgin (which }\end{array}$ & $\begin{array}{l}\text { breviations } \\
\text { aries language (Gamilaraa } \\
\text { Lake Macquarie language } \\
\text { taruah]) } \\
\text { Coast language (Warrimay } \\
\text { includes words borrowed }\end{array}$ & $\begin{array}{l}\text { y etc.) } \\
\text { (sometimes called 'Awaba } \\
\text { [Worimi], Guringay [Gring } \\
\text { from remote Aboriginal la }\end{array}$ & $\begin{array}{l}\text { kal'; includes } \\
\text { ai] etc.) } \\
\text { nguages) }\end{array}$ \\
\hline $\begin{array}{l}\text { Source abbreviatio } \\
A=\text { Ash, Giacon } \\
D=\text { Dixon, Ramso } \\
\text { L06 = Lissarrague } \\
\text { L10 = Lissarrague } \\
\text { (These abbreviatio }\end{array}$ & $\begin{array}{l}\text { ns } \\
\text { and Lissarrague } 2003 \\
\text { on and Thomas } 1990 \\
2006 \\
2010 \\
\text { ns are followed by the rele }\end{array}$ & vant page number and pla & aced in brackets.) \\
\hline $\begin{array}{l}\text { Glosses } \\
\text { The glosses follo } \\
\text { have been made }\end{array}$ & closely as & given in th & Iall emendations \\
\hline
\end{tabular}

Note A: The first two columns incorporate my transcription of the original list. The heading lines (here in italics) of these columns are type-printed on the original form. The two lines under the topmost heading are printed examples, not part of Coady's wordlist, which is hand-written. The two right-hand columns are, respectively, my interpretation of the etymologies, and the locations (where known. Some of these are speculative.) The conventions used in column 3 (the etymologies) are explained at the end of the list.

Note B: On the naming of Muswellbrook and the connection with mussels, see: http://www.muswellbrook. nsw.gov.au/About-Muswellbrook-Shire/History/Muswellbrook/Naming-muswellbrook.htm (accessed 14 September 2011).

Note C: According to McLellan (n.d.: 17), the Indigenous 'name for the area, Murrumdoorandi is now Murrurundi. The word particularly refers to the five rocks in the vicinity of the present town of which there are now four, a fifth having fallen since white men came into the area. Interpretation of the word is disputed, the favoured one is "the meeting place at the five fingers (rocks)".' 


\section{Analysis of the Scone list}

Even a cursory glance at the results of this tabulation indicates that the great majority of the words on the list pertain to a dialect of the Darling Tributaries language (which includes Gamilaraay). The figures are as follows: of the 78 items on the list, 60 (77 per cent) may be attributable to the Darling Tributaries language, and 38 of these (49 per cent of the total) are convincing; 12 (15 per cent) may be attributable to the Hunter River-Lake Macquarie language, and eight (10 per cent of the total) of these are convincing; six ( 8 per cent) may be attributable to the Lower North Coast language, but only one ( 1 per cent of the total) of these is convincing. ${ }^{21}$

Even if these figures are somewhat slippery (as a result, for example, of overlapping attributions where two or more languages use the same morpheme) the pattern is clear enough: the words on the list belong predominantly to the Darling Tributaries language. But what the list fails to provide is adequate information about the places these words were associated with, either as toponyms or simply as descriptors.

Certainly, the words were collated and transcribed at Scone, and a number of the recognisable names pertain to that region: Aberdeen; the junction of the Pages and Hunter Rivers (a short distance north-west of Aberdeen); possibly Moonan Flat, in the Upper Hunter; the Hunter River; ${ }^{22}$ Murrurundi; John Bingle's property 'Puen Buen' (on Dart Brook, west of Scone, according to Gray 1966); Gibbergunyah Creek (west of Scone); and possibly Muswellbrook, although this one is questionable. If the 'Mussel ground' on the list referred to Muswellbrook, one might have expected the author to be specific about this.

What of the other placenames that may have some association with this list? Gungal (if the word 'Gungalla' can be linked to it) is a little remote from this region, lying about $40 \mathrm{~km}$ due west of Muswellbrook (between Merriwa and Denman). And Boonoo Boonoo lies north of Tenterfield, more than $400 \mathrm{~km}$ north of Scone, in the region where Marbal appears to have been spoken. ${ }^{23}$

21 These figures add up to 77, not 78, because I have attributed 'Gibbagunyah' to Pidgin. The word giba does occur in both Gamilaraay (Ash, Giacon and Lissarrague 2003: 85) and LNC (Lissarrague 2010: 226); but its use in the compound form 'Gibbagunyah' suggests a borrowing from Pidgin rather than from one of the source languages. In the case of 'Mallegal', on the other hand, I have attributed it to HRLM on the basis of the presence of the suffix $-\mathrm{kal}$.

22 If 'Gingamboon' is indeed a name for the Hunter River, it seems likely that the river had different names at different points along its course. Wafer and Lissarrague (2008: 160, note 103) analyse four other names that have been attributed to it: 'Coquun', 'Myan', 'Coonanbarra' and 'Terrybong'.

23 See Wafer and Lissarrague (2008: 333). The 'Goonabil' on the Scone list is unlikely to refer to Coonamble (see Ash, Giacon and Lissarrague 2003: 95, 237), with which name it is undoubtedly cognate. Presumably the writer would have used the spelling 'Coonamble' if that is what he or she meant. 
Whatever the reasons (and several could be adduced) for the appearance of these two (apparent) placenames in the list, if we treat them as anomalies this at least allows us to make a statistical comparison. Eight of the ten words that can be plausibly linked to places are associated with locations between Murrurundi and Muswellbrook; only two of the words are associated with places outside this region. If we apply the figure of 80 per cent to the items on the wordlist, this suggests that something like 62 of the words on the list refer to places in the Murrurundi to Muswellbrook region, or to vocabulary items that were used there.

We can take this procedure a step further by considering the language associations of the eight placenames in question. One of them (Gibbergunyah Creek) is derived from NSW Pidgin. The other seven can be linked to the Darling Tributaries language: three of them convincingly, three plausibly, and one speculatively.

This is not to say that there may not have been placenames derived from either HRLM or LNC in the region between Murrurundi and Muswellbrook. But the weight of evidence suggests clearly that the majority language used in this region at the time the Scone list was collected was a dialect of the Darling Tributaries language: possibly Gamilaraay, or at least a closely related dialect. For present purposes, I shall call it the 'Upper Hunter dialect'.

\section{The language of the Upper Hunter}

In other words, Tindale's assertion that the 'Geawegal' occupied this region appears much less convincing than the original information about their location given by Fison and Howitt. There is further support for the view that the Darling Tributaries language was spoken in this region in an article by Albert MacDonald concerning 'The Aborigines on the Page and the Isis', published in the Journal of the Anthropological Institute in 1878.

The Pages River is a tributary of the upper Hunter, flowing from the Liverpool Range, past Murrurundi and Gundy, to join the Hunter not far upstream from Aberdeen. The Isis River is a tributary of the Pages River, and the confluence is a short distance upstream from Gundy. MacDonald gives an account of a group who 'held their boras' at a spot 'near the junction of the Rivers Page and Isis ... not far from the town of Aberdeen' (1878: 255). He notes that this group spoke "'Kamilaroi," varying slightly from that of the Namoi and Barwon' (1878: 257). This is substantiated by the small samples of language material he gives. He calls the people of this place 'the Murri', and mari is the Gamilaraay word for 'man' or 'Aboriginal person' (Ash, Giacon and Lissarrague 2003: 109). He 
also gives a couple of other words, and a song. This material is quite clearly either Gamilaraay or a closely related dialect with numerous cognates, ${ }^{24}$ and demonstrably not HRLM or LNC.

It is not clear how far down the Hunter Valley the country of these 'Murri' extended. But if they occupied the region between Murrurundi and Aberdeen, this would include the whole length of the Pages and Isis Rivers, and would also be consistent with my analysis of the toponymic data in the Scone wordlist.

There remains the question of whether the country in which the Upper Hunter dialect was spoken reached any further south than Aberdeen. The only placename on the Scone list that could provide any clues here is the one that is translated as 'Mussel ground' - and I have mentioned above that the link between this name and Muswellbrook is dubious. But I note in this connection that the title of a recent book about the Aboriginal history of Muswellbrook is drawn from the languages of the Kamilaroi and Wanaruah people who walked these lands' (Blyton, Heitmeyer and Maynard 2004: 2).

What this suggests is that Muswellbrook (just south of Aberdeen) may have been a transitional zone, or the southern limit of a transitional zone, between speakers of the Upper Hunter dialect and Wanarruwa (which is a dialect of HRLM). There is perhaps further confirmation of a meeting, perhaps even mixing, of languages in this region, or even further south, in the pen-name adopted by the same G.W. Rusden who provided the information for Fison and Howitt's account of the 'Geawegal'.

The name under which Rusden published the second edition of his epic poem on Aboriginal themes entitled 'Moyarra' (Rusden 1891) - and various other works - was 'Yittadairn'. As mentioned in an earlier note, 'Rusden was identified with it [the Geawegal 'tribe'], and spoke the language as a youth' (Fison and Howitt 1880: 279). But the components of this pseudonym could perhaps be analysed as yitirr (HRLM: 'name'; Lissarrague 2006: 146) + dhayn (DT: '(Aboriginal) man'; Ash, Giacon and Lissarrague 2003: 60). In other words, this nom de plume possibly has a mixed etymology. ${ }^{25}$

Brayshaw, in her foundational study of Aborigines of the Hunter Valley (1986) writes that 'the Wonaruah and the Geawegal and probably the Gringai in the central and upper Hunter were part of the "Kamilaroi nation"" (1986: 51). It is

24 John Giacon (pers. comm. 24 October 2006) observes as follows: 'there is a lot here that looks like Gamilaraay, so either it is Gamilaraay or a language with lots of cognates... The song looks as if it is about someone who is sick getting better - there are lots of Gamilaraay words, but I can't be all that sure about the overall meaning.'

25 The same may be true of the names of the various characters in 'Moyarra' (Koreungat, Moyarra, Muntookan, Myta, Warrawe), although they are hard to analyse, because of the absence of glosses. Nonetheless, they give strong hints of being a mixture of the three languages I have been dealing with in this paper. On Rusden, see Blainey and Lazarus (1976). 
unclear how this conclusion has been arrived at, but it is probably based on the fact that the Wanarruwa, Kayawaykal and Guringay all used a section system in which the section names were cognate with those in Gamilaraay. This is certainly an indication of some kind of social relationship, which undoubtedly included intermarriage. But I suggest it is not sufficient to include Wanarruwa, Kayawaykal and Guringay as members of the Gamilaraay 'nation'. Linguistically, Wanarruwa, and - as I have tried to show - probably also Kayawaykal, belong with other dialects of the Hunter River-Lake Macquarie language, while Guringay belongs with the other dialects of the Lower North Coast language.

A discrepancy between linguistic affiliations and social affiliations is, of course, never out of the question, so I do not discount the possibility that the Wanarruwa, Kayawaykal and Guringay had a closer social relationship with the Gamilaraay than they had with speakers of dialects more closely related to their own. But this cross-cutting pattern of relationships is more an indication of the relative autonomy of local dialect groups, rather than evidence for their membership in some superordinate grouping such as a 'nation' ${ }^{26}$

\section{The landnám question}

The only argument that could potentially save Tindale's attribution of the Upper Hunter region to 'Geawegal' would be one based on landnám. This would mean that the region had been inhabited originally by speakers of Kayawaykal, but the latter were succeeded at some point by speakers of the Upper Hunter dialect, who engaged in a process of renaming. In other words, one way of explaining the scattering of placenames on the Scone list that are possibly derived from HRLM would be as evidence of a prior linguistic stratum.

The term landnám comes from the mediaeval Landnámabók, 'The Book of the Settlement of Iceland'. Two Australian writers have glossed the expression as 'land-taking and land-naming' (Leer 1985: 11; Gilbert 1998: 16), and this captures the polysemous nature of the word nám. ${ }^{27}$ Although it does not mean 'naming', but rather 'taking', this is not just 'taking' in a literal sense, but also in the sense of 'grasping with the mind'. In fact, one of its meanings is 'learning, study' (Zoëga 1910: 309-310).

\footnotetext{
26 There is a different aspect of this question that other researchers may wish to follow up, namely, whether the languages dealt with in this paper have some demonstrable relationship to the ecology of the region. There is a tantalising clue in the fact that the Upper Hunter is included in the Central Western Slopes (botanical) Subdivision, even though it lies to the east of the Great Dividing Range (Curran, Clark and Bruhl 2008: 385. See their bioregion map [fig. 1 on p. 383] for a clear visual representation). In other words, it appears that the vegetation, like the Darling Tributaries language, spills over the Range into the Upper Hunter.

27 My understanding is that nám is the nominative singular form of this (strong, neuter) noun, and náma the genitive plural. I am relying here on Zoëga's A Concise Dictionary of Old Icelandic (1910).
} 


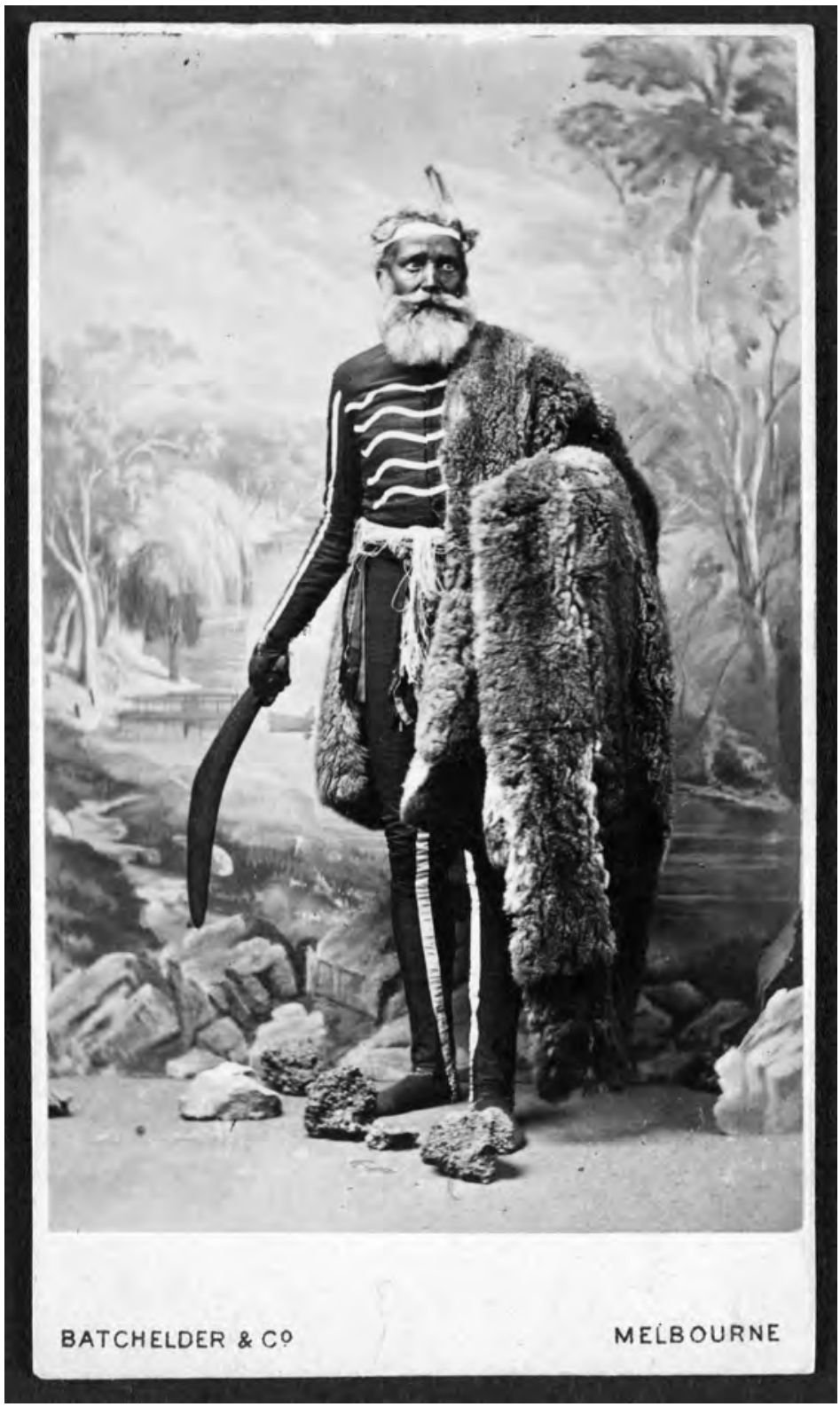

\section{Image 1: G.W. Rusden dressed as 'Yittadairn'.28}

Source: Pictures Collection, State Library of Victoria.

28 'Yittadairn' was, as mentioned above, the pen-name used by G.W. Rusden for some of his publications. This photograph on carte-de-visite mount is entitled 'G. W. Rusden as Yittadairn' in the catalogue of the State Library of Victoria (http://handle.slv.vic.gov.au/10381/111666, accessed 5 July 2013), from whose copy the version here is reproduced. The annotation in the catalogue of the Australian National Library quotes from the auction documentation: 'An extraordinary image of Rusden, the historian of Australia and New Zealand 
Fick, Falk and Torp trace it to the proto-Germanic root "nem (1909: 293), one of the glosses for which they list as geistig aufnehmen ('take in intellectually'). Campbell gives a sense of the semantic scope of landnám when he explains it as 'the method of acquisition of ... territory ... not by prosaic physical action, but poetically, by intelligence and the method of art' (1986: 34).

Typically, landnám entails two kinds of naming processes. One is the adoption of toponyms used by the previous inhabitants; the other is the introduction of new toponyms in the immigrants' own language, often derived from placenames used in their former place of residence.

Campbell implies that landnám is a cultural universal (giving as examples the myths of the Hebrews, the Vedic Aryans and the Navaho). But this is problematic in the context of Australia, since it is generally assumed that only non-Indigenous Australians engage in the practice. For Aboriginal people, 'since placenames are part of the Law (yumi) assigned by Dreamings (mangaya, puwarraj) to a specific place, mere human beings cannot lift those names up and drop them in other places' (McConvell 2002a: 52).

This apparent difference is perhaps merely a reflection of the different methods - and also time-scale - of landnám as practised by Indigenous and non-Indigenous Australians. ${ }^{29}$ Presumably the first humans to arrive on these shores, whether they came in one or several waves, went through a process of 'land-taking and land-naming' as they spread throughout the continent; and the same process would no doubt have been necessitated by internal migrations resulting from the various climatic and geographical changes that the island has undergone since. So it would be surprising if landnám were not still practised, at least to some minor degree, in Aboriginal Australia - for example, in cases of succession to a particular tract of land (cf. McConvell 2004: 42). The fact that this is rationalised in terms of the ideology of 'the Dreaming' simply obscures the political processes involved.

\footnotetext{
dressed in an elaborate black fleshing costume, holding a boomerang and in a possum skin or Kangaroo rug' (http://nla.gov.au/nla.pic-vn4223009, accessed 17 September 2011). I have included a mention of this photograph in the list of references under the name of the photographers, 'Batchelder \& Co.'.

29 Some good examples of the rather indiscriminate use by non-Indigenous Australians of Aboriginal placenames are provided by the Scone list itself. In recent times, one word (Galliebarinda) has been applied to a waterfall in the Otway Ranges, in Victoria, and another (Gillebri) to a property near St George, in Queensland. Other words on the list coincide with the names of a town (Bambill), a mountain (Moonbil), pastoral stations (Goonabil, Marooan, Woolamon, Neemin, Gungalla), a cattle company (Boogal), streets (Boombil, Billanbri, Walgooan), houses or homesteads (Bareemal, Marooan), a ship (Goorangi), a yacht (Moonbil), racehorses (Boogal, Goonabil, Moonbil) and a greyhound (Gibbergunyah). The spellings are identical to those on the Scone list except in the cases of 'Goorangi', which was a commonly used variant of the ship's correct name, 'Goorangai'; and of 'Gibbergunyah', which is in any case a Pidgin word with variable spellings. These usages come from various parts of Australia that have no obvious connection to the Upper Hunter. To thread a way through this labyrinth of possible borrowings is beyond the scope of the present paper.
} 
Unfortunately, although there have been advances in what McConvell has called 'linguistic stratigraphy' in some parts of Australia (see e.g. McConvell 2002b, 2004), the Hunter does not yet fulfill the basic requirement of 'historical analysis on all or most of the languages in the region concerned' (McConvell 2002b: 262). In other words, it is too early to make any kind of case based on landnám for the area under consideration here. The mixture of languages reflected in the Scone list could, perhaps, be taken as an indication of an earlier process of landnám; but there are other, more plausible, explanations, too various to enumerate in any detail. ${ }^{30}$ To conclude, the evidence, at this point in time, appears to demonstrate that the region attributed by Tindale to the 'Geawegal' was in fact occupied by speakers of a dialect - which I call the 'Upper Hunter dialect' - of the Darling Tributaries language.

\section{References}

Aboriginal Languages Research and Resource Centre n.d., 'Map of NSW Aboriginal languages': http://ab-ed.boardofstudies.nsw.edu.au/bosImg window.cfm?objectid=03540C9F-F074-1B07-416F6CDE53A110F7 or alternatively http://www.tne.edu.au/ /media/images/website_images/Misc/ NSWlanguagesmap.ashx (both accessed 6 April 2014).

Anonymous ca 1845, Vocabulary of the Allyn River Black's [sic] language, MS held by the Mitchell Library, State Library of NSW, Sydney (ML Aa52/1-3; CYReel 2355, frames 88-115).

Ash, A., J. Giacon and A. Lissarrague (eds) 2003, Gamilaraay, Yuwaalaraay \& Yuwaalayaay Dictionary, IAD Press, Alice Springs.

Batchelder \& Co. ca 1880, 'G. W. Rusden as Yittadairn', photograph on carte-devisite mount, Batchelder \& Co., Melbourne. Reproduced here from the copy held by the State Library of Victoria, which has made the image available online at http://handle.slv.vic.gov.au/10381/111666 (accessed 27 July 2013).

Blainey, A. and M. Lazarus 1976, 'Rusden, George William (1819-1903)', Australian Dictionary of Biography, National Centre of Biography, ANU: http://adb.anu.edu.au/biography/rusden-george-william-4523/text7405 (accessed 14 September 2011). First published in Australian Dictionary of Biography, vol. 6, Melbourne University Press, Melbourne.

Blyton, G., D. Heitmeyer and J. Maynard 2004, Wannin Thanbarran: A History of Aboriginal and European Contact in Muswellbrook and the Upper Hunter

30 It could, for example, be a result of what McConvell calls 'the normal process of contact and diffusion' (2002b: 263), possibly speeded up by forced displacement of Aboriginal people by the European colonisers. 
Valley, Umulliko Centre for Indigenous Higher Education, University of Newcastle, and Muswellbrook Shire Council Aboriginal Reconciliation Committee, Muswellbrook.

Brayshaw, H. 1986, Aborigines of the Hunter Valley: A Study of Colonial Records, Scone \& Upper Hunter Historical Society, Scone.

Campbell, J. 1985, The Inner Reaches of Outer Space: Metaphor as Myth and as Religion, A. van der Marck Editions, New York.

Central Mapping Authority, NSW Department of Lands ('CMA') 1987, 'Aboriginal New South Wales', Central Mapping Authority, Bathurst.

Coady, J. 1899, 'Scone' (return from Senior Sergeant John Coady, Police Station, Scone, of 16/10/1899), pp. 297-299, microfilm roll 1, frames 010328-010330). In 'Aboriginal names. "Collection of Native Names of places with their meanings."' Circular distributed to police stations in New South Wales, 16/8/1899, by the Anthropological Society of Australasia. Returns paginated and indexed. Microfilmed by W \& F Pascoe Pty Ltd 1991, under the title 'Anthropological Society of Australia'. Roll 1 (of 5 rolls). Digital version made available by Geographical Names Board of NSW. CD Rom held by AIATSIS library at 910.3009944 GEO C-D. (Geographical Names Board of New South Wales [2003], 'Royal Anthropological Society of Australasia manuscripts dated 1900'. Microfilm has 476+456+949+449+1358 frames as reproduced in the 5 rolls on the CD.) Original documents now held by the Mitchell Library, State Library of NSW, Sydney. Catalogued under 'Royal Anthropological Society of Australasia - Records, 1885-1914, with additional material, 1921-1926, ca. 1991, and papers of Alan Carroll, 1886-1892' (MLMSS 7603). For further details see: http://acms.sl.nsw.gov.au/item/itemDetailPaged. aspx?itemID=421736 (accessed 16 September 2011). Scone list included in microfilm CY 4555, frames 1-495.

Curran, T. J., P. J. Clarke, and J. J. Bruhl 2008, 'A broad typology of dry rainforests on the western slopes of New South Wales', Cunninghamia 10(3): 381-405: http://www.une.edu.au/ers/staff-profile-doc-folders/peter-clarke/2008curran-et-al-cunninghamia.pdf (accessed 5 July 2013).

Dixon, R.M.W., W.S. Ramson and Mandy Thomas 1990, Australian Aboriginal Words in English: Their Origin and Meaning, Oxford University Press, Melbourne.

Enright, W. J. 1932, 'The Kattang (Kutthung) or Worimi: an Aboriginal tribe', Mankind 1(4): 75-77.

Fawcett, J.W. 1898, 'Customs of the Wannah-Kuah [sic] tribe, and their dialect or vocabulary', Science of Man 1(8): 180-181. 
Fick, A., H. Falk and A. Torp 1909, Vergleichendes Wörterbuch der indogermanischen Sprachen, dritter Teil: Wortschatz der germanischen Spracheinheit, Vandenhoeck \& Rupprecht, Göttingen. Electronic version created by Sean Crist, 2003: http://www.ling.upenn.edu/ kurisuto/ germanic/pgmc_torp_about.html (accessed 14 September 2011).

Fison, L. and A.W. Howitt 1880, Kamilaroi and Kurnai, George Robertson, Melbourne. (Appendix G: 'The Geawe-gal Tribe', pp. 279-284.)

Fraser, J. (ed.) 1892, An Australian Language as Spoken by the Awabakal, Charles Potter, Government Printer, Sydney.

Gilbert, H. 1998, Sightlines: Race, Gender, and Nation in Contemporary Australian Theatre, University of Michigan Press, Ann Arbor.

Gray, N. 1966, 'Bingle, John (1796-1882)', Australian Dictionary of Biography, National Centre of Biography, ANU: http://adb.anu.edu.au/biography/binglejohn-1780/text2001 (accessed 12 September 2011). First published in Australian Dictionary of Biography, vol. 1, Melbourne University Press, Melbourne.

Hale, H. E. 1846, 'Ethnography and philology', volume 6 of United States Exploring Expedition, during the Years 1838, 1839, 1840, 1841, 1842, under the Command of Charles Wilkes, U. S. N., Lea and Blanchard, New York.

Horton, D. R. (comp.) 1996, 'Aboriginal Australia' (map produced to accompany David Horton (ed.), Encyclopaedia of Aboriginal Australia), Aboriginal Studies Press for the Australian Institute of Aboriginal and Torres Strait Islander Studies, Canberra.

Howitt, A.W. 1904, The Native Tribes of South-East Australia, Macmillan, London.

Leer, M. 1985, 'At the edge: geography and the imagination in the work of David Malouf', Australian Literary Studies 12(1): 3-21.

Lissarrague, A. 2006, A Salvage Grammar and Wordlist of the Language from the Hunter River and Lake Macquarie, Muurrbay, Nambucca Heads.

- 2010, A Grammar and Dictionary of Gathang: The Language of the Birrbay, Guringay and Warrimay, Muurrbay, Nambucca Heads.

MacDonald, A. C. 1878, 'The Aborigines on the Page and the Isis', Journal of the Anthropological Institute of Great Britain and Ireland 7: 255-258.

McConvell, P. 2002a, 'Changing places: European and Aboriginal styles', in The Land is a Map: Placenames of Indigenous Origin in Australia, Luise Hercus, Flavia Hodges and Jane Simpson (eds), Pandanus Books in association with Pacific Linguistics, Canberra: 50-61. 
- 2002b, 'Linguistic stratigraphy and native title: the case of ethnonyms', in Language in Native Title, John Henderson and David Nash (eds), Aboriginal Studies Press, Canberra: 259-290.

- 2004, 'A short ride on a time machine: linguistics, cultural history and native title', in Crossing Boundaries: Cultural, Legal, Historical and Practice Issues in Native Title, Sandy Toussaint (ed.), Melbourne University Press, Carlton: 34-49.

McLellan, A.A. n.d., Thomas Haydon of Bloomfield, Blandford, Advocate Print, Quirindi.

Miller, J. 1985, Koori, a Will to Win: The Heroic Resistance, Survival and Triumph of Black Australia, Angus and Robertson, London.

Miller, R. 1887, 'No. 188: the Hunter River. The Wonnarua tribe and language', in The Australian Race, E.M. Curr (ed.), John Ferres, Government Printer, Melbourne: vol. 3 (of 4), pp. 352-359.

Muswellbrook Shire Council, n.d., 'The naming of our town as "Muswellbrook", http://www.muswellbrook.nsw.gov.au/About-Muswellbrook-Shire/History/ Muswellbrook/Naming-muswellbrook.htm (accessed 14 September 2011).

Oppliger, M. 1984, 'The phonology and morphology of Awabakal: a reconstitution from early written sources', BA (Hons) thesis, University of Sydney.

Rusden, G.W. (writing as 'Yittadairn') 1891, Moyarra: An Australian Legend in Two Cantos, E.A. Petherick \& Co., London, Melbourne \& Sydney.

Threlkeld, L. E. 1834, An Australian Grammar, Stephens and Stokes, Sydney.

Tindale, N. B. 1974, Aboriginal Tribes of Australia, University of California Press, Berkeley, and ANU Press, Canberra. (Includes map, 'Tribal boundaries in Aboriginal Australia'.)

Wafer, J., and A. Lissarrague 2008, A Handbook of Aboriginal Languages of New South Wales and the Australian Capital Territory, Muurrbay, Nambucca Heads.

- 2011, 'The Kuringgai puzzle: languages and dialects on the NSW Mid Coast', in Indigenous Language and Social Identity: Papers in Honour of Michael Walsh, Brett Baker, Ilana Mushin, Mark Harvey \& Rod Gardner (eds), Pacific Linguistics, Canberra: 155-168.

Zoëga, G. T. 1910, A Concise Dictionary of Old Icelandic, Clarendon Press, Oxford. 
This text taken from Indigenous and Minority Placenames: Australian and International Perspectives, Edited by Ian D. Clark, Luise Hercus and Laura Kostanski, published 2014 by ANU Press, The Australian National University, Canberra, Australia. 